Per quanto riguarda finalmente il confronto delle figure 4 e 5 noi possiamo riferirci all'analisi esposta nell'art. 8, dalla quale emerge ehe esse sono nelle loro minime parti similmente ovvero inversamente poste, secondo che si sceglie la prima o la seconda soluzione, cioè secondo che si pone

orvero

$$
\mathbf{P}+i \mathrm{Q}=f(p+i q) \quad \text { e } \quad \mathrm{P}-i \mathrm{Q}=f^{\prime}(p-i q)
$$

$$
\mathbf{P}+i \mathrm{Q}=f(p-i q) \quad \text { e } \quad \mathbf{P}-i \mathrm{Q}=f^{\prime}(p+i q) .
$$

Da tutto ciò ricaviamo finalmente la conclusione, che se la rappresentazione sulla superfieie definita dall'equazione $\Psi=0$ deve essere nelle sue parti infinitesime non solamente simile, ma altresi similmente posta rispetto alla figura originaria esistente sulla superficie $\psi=0$, bisogna aver riguardo al numero delle quantità negative che si presentano fra le quattro seguenti :

$\frac{a b^{\prime}-b a^{\prime}}{h},\left(\frac{\mathrm{d} p}{\mathrm{~d} t}\right) \cdot\left(\frac{\mathrm{d} q}{\mathrm{~d} u}\right)-\left(\frac{\mathrm{d} p}{\mathrm{~d} u}\right) \cdot\left(\frac{\mathrm{d} q}{\mathrm{~d} t}\right), \quad\left(\frac{\mathrm{dP}}{\mathrm{dT}}\right) \cdot\left(\frac{\mathrm{d} Q}{\mathrm{~d} \mathbf{U}}\right)-\left(\frac{\mathrm{dP}}{\mathrm{d} \mathbf{U}}\right) \cdot\left(\frac{\mathrm{dQ}}{\mathrm{dT}}\right), \frac{\mathrm{AB}^{\prime}-\mathrm{BA}^{\prime}}{\mathrm{H}} ;$

se fra esse non compajono quantilà negative o se il numero di quelle che vi compajono è pari, bisogna prendere la prima soluzione; se invece le negative sono una o tre, dev'essere scelta la seconda soluzione. Altrimenti sussiste una similitudine inversa.

Del resto si può mostrare anche che, se si indicano ordinatamente con $r, s, \mathrm{~S}, \mathbf{R}$ Ie quattro quantità precedenti, si ha sempre

$$
\frac{r \sqrt{ }(e e+g g+h h)}{s}= \pm n, \quad \frac{\mathrm{R} \sqrt{ }(\mathrm{EE}+\mathrm{GG}+\mathrm{HH})}{\mathrm{S}}= \pm \mathrm{N}
$$

conservando ad $n, \mathrm{~N}$ il significato che hanno nell'art. 5; ma non ci soffermeremo a dare la dimostrazione, non difficile a trovarsi, di questo teorema, giacchè ciò non é punto necessario per lo scopo che abbiamo di mira.

\title{
PUBBLICAZIONI RECRNTI
}

Trud N. $\quad$ - Teoria de'determinanti, e loro applicazioni. Vol. in $9^{\bullet}$ Napoli 1862.

- Sull'inviluppo delle corde di costante grandezza nelle curve di $2^{\circ}$ grado(Rendiconto Accademia di Napoli. Maggio 1862).

Battaglini G. - Sopra alcune proprietà delle linee di $2^{\circ}$ grado. (Rendiconto Accademia di Napoli. Maggio 1862.

- Nota sulle superficie di Secondo Grado. (Rendiconto Accademia di Napoli. Giugno 1862).

- - Nota sui determinanti. (Rendiconto Accademia di Napoli. I.uglio 1862)

Fergola E. - Sulla risoluzione per serie delle equazioni trinomie di grado qualunque. (Rendiconto Accademia di Napoli. Maggio 1862).

Rubini R. - Elementi di Algebra in $8^{\circ}$. Napoli 1862.

Pievani Dr. Ant. - Circa le espressioni, che ponno essere sviluppate secondo derivate lineari d'ordine crescente positivo, ed intero di una medesima funzione. Milano 1862. Fascicolo in foglio di $\mathbf{4 3}$ pagine.

Poncelet J.-V. - Applications d'analyse, et de géometrie qui ont servi, en 1822, de principal fondement au traité des propriétés projectives des figures. Vol. in $8^{\circ}$. Paris 1862.

Chasles

- Lettre à M. L. Am. Sédillot, sur la question de la Variation lunaire découverte par Aboul-Wefa, - 15 pag. Paris 1862 .

WEIERSTR ASS

WEIERSTRASS (Monastbericht der K. Akad. der Wiss. zu Berlin, Februar 1862.

(Monastbericht der K. Akad. der Wiss. zu Berlin, Februar 1862.
- Studien tiber die Integration linearer Differential. Gleichungen in $8^{\circ}$. Wien 1862. 